\title{
30-Day Potentially Preventable Hospital Readmissions In Older Patients: Clinical Phenotype And Health Care Related Risk Factors
}

This article was published in the following Dove Press journal: Clinical Interventions in Aging

\author{
Valeria Calsolaro $\mathbb{D}^{1, *}$ \\ Rachele Antognoli ${ }^{1, *}$ \\ Giuseppe Pasqualetti' \\ Chukwuma Okoye' \\ Ferruccio Aquilini ${ }^{2}$ \\ Michele Cristofano ${ }^{2}$ \\ Silvia Briani ${ }^{2}$ \\ Fabio Monzani (D) \\ 'Geriatrics Unit, Department of Clinical \\ and Experimental Medicine, University \\ Hospital of Pisa, Pisa, Italy; ${ }^{2}$ Health \\ Management Department, University \\ Hospital of Pisa, Pisa, Italy \\ *These authors contributed equally to \\ this work
}

Correspondence: Fabio Monzani Geriatrics Unit, Department of Clinical \& Experimental Medicine, University Hospital of Pisa, via Paradisa 2, Pisa 56124, Italy

Tel +393337733135

Email fabio.monzani@med.unipi.it
Purpose: Early readmission rate has been regarded as an indicator of in-hospital and postdischarge quality of care. Evaluating the contributing factors is crucial to optimize the healthcare and target the intervention. In this study we evaluated the potential for preventing 30-day hospital readmission in a cohort of older patients and identified possible risk factors for readmission.

Patients and methods: Diagnosis-Related Group (DRG) codes of patients consecutively hospitalized for acute disease in the Geriatrics Unit of the University Hospital of Pisa within a 1-year window were recorded. All the patients had received a comprehensive geriatric assessment. Crossing and elaboration of the DRG codes was performed by the Potentially Preventable Readmission Grouping software ( $3 \mathrm{M}^{\mathrm{TM}}$ Corporation). DRG codes were classified as stand-alone admissions (SA), index admissions (IA) and potentially preventable readmissions (PPR) within a time window of 30 days after discharge.

Results: In total, 1263 SA and 171 IA were identified, with an overall PPR rate of $11.9 \%$. Hospitalizations were significantly longer in IA and PPR than SA $(p<0.05)$. The more frequent readmission causes were acute heart failure, pulmonary edema, sepsis, pneumonia and stroke. In acute heart failure a nonlinear U-shaped readmission trend (with nadir at 5 days of hospitalization) was observed while, in all the other DRG codes, the PPR rate increased with increasing length of hospitalization. Comprehensive geriatric assessment showed a significantly lower degree of disability and comorbidity in SA than IA patients. At stepwise regression analysis, a high degree of disability and comorbidity as well as the diagnosis of sepsis emerged as independent risk factors for PPR.

Conclusion: Addressing PPR is crucial, especially in older patients. The adequacy of treatment during hospitalization (especially in cases of sepsis) as well as the setting of a comprehensive discharge plan, accounting for comorbidity and disability of the patients, are essential to reduce PPR.

Keywords: potentially preventable readmission, length of stay, multidimensional geriatric assessment, older patients, frailty

\section{Introduction}

Early readmissions represent an ongoing and worldwide challenge in clinical settings, being a huge social and economic burden, especially in older people. ${ }^{1-3}$ One of the first studies on readmissions, which can be seen as an indicator of both prognosis and quality of care, was done in the UK, evaluating hospital readmissions within 28 days from discharge over 17 years. The readmission rate, in elective and emergency settings, increased in 1985 compared to 1968, possibly because of the 
shorter length of stay and managerial reasons. ${ }^{4}$ One of the possible reasons of early readmission has been identified as poor management of early follow-up after discharge. ${ }^{2}$ In addition to poorer health status and chronic diseases, ${ }^{5}$ markers of frailty and disability have been identified as risk factors for readmission in an older population. ${ }^{6}$ That was confirmed in a systematic review, which identified morbidity and disability as risk factors for readmission, together with prior admissions and duration of hospital stay. ${ }^{7}$ Considering the complexity of the geriatric patient and the negative impact of readmissions on the prognosis, a full exploratory evaluation of the older patients, comprehensive multidisciplinary approach and post-discharge plan, is necessary to address the main risk factors for readmission and reduce readmissions themselves. The aim of the current study was to evaluate the 30-days readmissions in a cohort of geriatric patients consecutively discharged from the Geriatrics Unit of the University Hospital of Pisa, from 1 July 2016 to 30 June 2017. The major endpoint of the study was to identify potentially preventable readmissions (PPR) by using either powerful clinical grouping logic (Potentially Preventable Readmission Grouping software, $3 \mathrm{M}^{\mathrm{TM}}$ Corporation) or comprehensive geriatric assessment, aiming to target areas for potential improvement of care quality, and to identify predictive factors of readmission. We also compared the readmitted patients with the ones admitted only once, without any re-entry within 30 days from discharged ("stand-alone" admissions, SA). We hypothesized that addressing potentially preventable readmissions, especially in the oldest old population, could identify patients who need early follow-up and a comprehensive discharge plan, accounting for the disability and comorbidity of the patient. We believe it is essential that policymakers seek input from real world data to improve collaboration between administrators, clinicians and information technology specialists for a useful transition between emergency and longitudinal care.

\section{Materials And Methods}

\section{Cases' Selection}

All the patients consecutively admitted for acute disease and discharged from the Geriatrics Unit between July 2016 and June 2017 were enrolled. At admission, according to the University Hospital of Pisa policy, each patient gave written informed consent to anonymous use of clinical data; informed consent was provided by the legally authorized delegate in cases of patients who were temporarily or permanently without capacity. The study protocol was approved by the Institutional Ethics Committee. The 30-days re-admissions from July 2016 onward were retrospectively analyzed crossing the data collected from the Hospital Discharge Register containing the DiagnosisRelated Group (DRG) codes. Patients discharged from the Geriatrics Unit and readmitted in any surgical or rehabilitation unit, deceased patients and those discharged against medical advice were excluded. Non-preventable readmissions, related to advanced and metastatic malignancy, poly-trauma, burns, psychiatric diseases, abuse substances and palliative care were also excluded, since the condition itself is a risk factor for multiple admissions, while our focus was on acute disease. The above-mentioned conditions were either identified in the medical history, or newly diagnosed.

\section{Admissions' Classification Protocol}

Crossing and elaboration of the DRG codes were performed by the potentially Preventable Readmission Grouping software $\left(3 \mathrm{M}^{\mathrm{TM}}\right.$ Corporation, Salt Lake City, Utah USA). The key was to determine which readmissions were clinically preventable. The $3 \mathrm{M}^{\mathrm{TM}}$ software identifies Potentially Preventable Readmissions (PPR) based on clinically precise criteria. It determines whether a readmission is clinically related to a prior one based on the patient's diagnosis and procedures in the prior admission and the reason for readmission. Thus, the Index Admissions (IA) represent the initial admissions generating the chain of PPR. Conversely, hospitalizations were considered "stand-alone" (SA) when the cause of the subsequent admissions was not related to the prior admissions, and not potentially preventable.

The $3 \mathrm{M}^{\mathrm{TM}}$ software uses clinical logic to determine the likelihood that a readmission could be clinically related to a prior admission and excludes prior admissions for clinically unrelated conditions such as trauma, cancer and burns. The software operates according to the All-Patient-RefinedDiagnosis-Related Group (APR-DRG) code, in order to stratify the admissions and verify the connection between the initial and the subsequent admissions. The APR-DRG code system is comprehensive of subclasses of progressive severity and defines the burden of clinical severity, quantified as the resources employed in the course of the hospital care. In detail, a matrix composed by 314 rows for the APR-DRG codes of the candidate admissions and 314 columns for the APR-DRG codes of the subsequent admissions was 
generated to identify the chain of IA and related PPR. Each of the 98,596 matrix cells represents a unique combination of the burden of the resources employed in the course of the initial and the following admissions. This matrix system was validated by a panel of specialists, who examined at least twice each of the 98,596 cells and identified 32,230 cells $(33 \%)$ as the minimum cut off for assessing the relationship between IA and PPR. In computing the PPR rate, the numerator is the number of PPR chains and the denominator the number of candidate admissions. Examples of PPR are defined by one or more factors as follows: i) prosecution or repetition of the same cause or a condition strictly related to IA; ii) acute worsening of a chronic condition potentially following a therapy given during the IA or in the immediate follow-up; iii) surgical procedure to solve or prosecute the therapy set during the IA; iv) surgical procedure to solve an unpredictable complication of treatment set during the IA.

\section{Patients' Assessment}

The Geriatrics Unit of the University Hospital of Pisa admits only acute older patients from the emergency department (ED). All the patients received comprehensive geriatric assessment within 24 hours from admission, administered by trained geriatricians. Comprehensive geriatric assessment - exploring cognitive and functional areas, nutritional status and comorbidities — was obtained using the following geriatric scales: Cumulative Illness Rating Scale (CIRS), Activities of Daily Living (ADL), and Instrumental Activities of Daily Living (IADL) (as referred before hospitalization), Short Portable Mental Status Questionnaire (SPMSQ), Mini Nutritional Assessment (MNA) [categorized in dichotomous variables (malnutrition or risk of malnutrition and normal)], and the Confusion Assessment Method (CAM). The results of comprehensive geriatric assessment were recorded in a database together with demographic data, length of hospital stay, and discharge diagnoses.

\section{Statistical Analysis}

Statistical analysis was performed by using SPSS 21.0 statistical software package (IBM Corporation, Armonk, NY, USA). Continuous variables are expressed as mean $\pm \mathrm{SD}$, ordinal variable as median and range and categorical variables as percentage. Normally distributed parameters were analyzed by $t$-test, while Kruskal-Wallis test was used for not normally distributed parameters. Spearman correlation was used for either continuous or dichotomous variables. Analysis of variance was used to compare continuous normally distributed variables, whereas the $\chi^{2}$ test was used for categorical or dichotomous variables. Stepwise backward multivariate logistic regression analysis, with significance limit at $\mathrm{p}<0.05$ for each covariate to be removed from the model, was also performed. The aim of the logistic regression model is to determine the odds ratios for any variable included into the model, and to display the predicted probability of a positive outcome for each observation in the data. The lowest and highest value of the covariate pattern represents the extreme values of the probability of early readmission (marginal risk). Statistical significance was assigned for $\mathrm{p}<0.05$.

\section{Results \\ Potentially Preventable Readmission Grouping Software Analysis}

We analyzed 1929 DRG codes. Among these, 289 (15\%) were excluded due to the following conditions: 143 had active cancer (metastatic or not), 137 died during admission, five went to other Units and four voluntarily resigned (Figure 1). The remaining 1640 admission codes were classified by the software as follows: 1263 SA (77.0\%), 171 IA (10.4\%), and 206 PPR (12.6\%). The 171 IA generated 206 PPR since 142 patients $(83.0 \%)$ were readmitted once, 24 $(14.0 \%)$ twice, four $(2.4 \%)$ three times and one $(0.6 \%)$ four times. Thus, the PPR rate (IA/SA+IAx100) was $11.9 \%$. Demographic data and length of hospital stay of the patients as classified by the software are described in Table 1. In detail, age was similar in the three groups (SA, IA and PPR), while the median length of hospital stay was significantly longer in the IA and PPR groups compared to the SA group (6 [2-14] vs 5 [4-6] days, $\mathrm{p}<0.05)$ (Table 1). The prevalence of men was slightly more frequent in the IA and PPR groups than in the SA group (45.6\% vs $44.6 \%$ ), although not reaching statistical significance. However, two distinct trends of PPR rate were observed according to DRG codes. In detail, the rate of re-hospitalization showed a $\mathrm{U}$ shape in DRG code 127 (acute heart failure), progressively increasing with the length of hospital stay either shorter and longer than 5 days while, in all the other DRG codes, a progressive rise of PPR rate with increasing length of hospitalization was observed (Figure 2).

The five most frequent DRG codes observed in the IA group, inducing 39.7\% of all PPR, are shown in Figure 3. Among them, DRG code 127 (heart failure) was associated with a significantly higher number of multiple readmissions (1.61 PPR generated by each IA) compared to the other four more frequent DRG codes (087 [pulmonary 
1929 DRG codes included in PPRG analysis

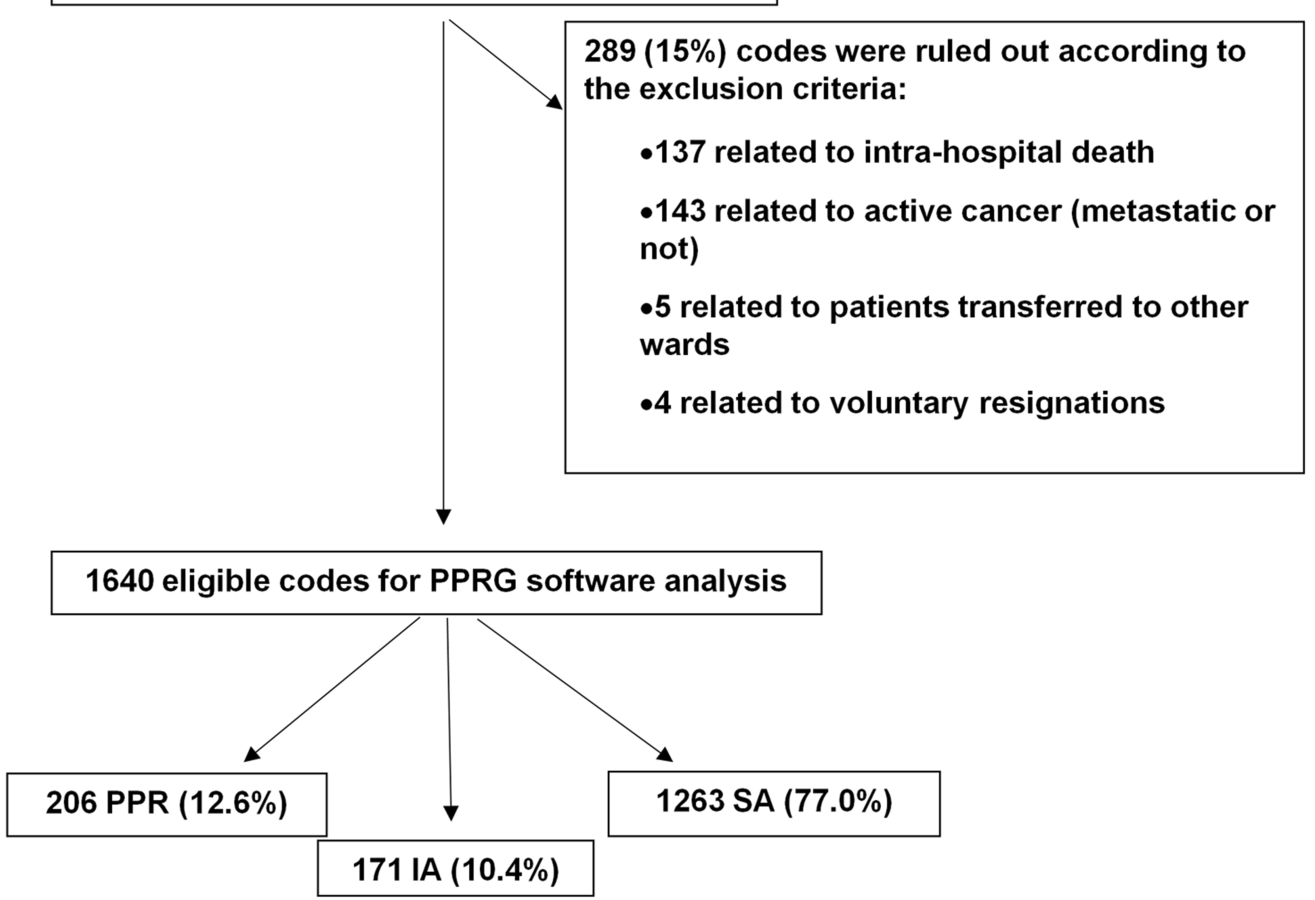

Figure I Flowchart of inclusion and exclusion criteria.

Abbreviations: DRG, Diagnosis Related Groups; PPR, Potentially Preventable Readmissions; PPRG, Potentially Preventable Readmissions Grouping; IA, Index Admission; SA, Stand Alone admissions.

oedema], 576 [sepsis], 089 [pneumonia], 014 [stroke]) that generated a mean of 1.2 PPR for each IA $(\mathrm{p}<0.05)$.

\section{Comprehensive Geriatric Assessment}

Almost three quarters (74.3\%) of the hospitalized patients were older than 80 years and showed a high burden of comorbidity (mean CIRS-C score 5.3) with moderate disability (mean ADL score 2.7; mean IADL score 2.3) and mild/moderate cognitive impairment (mean SPMSQ score 4.8) (Table 2). However, when comparing patients in the IA and SA groups significant differences in all the geriatric scale scores were obtained. In detail, patients in the IA group showed higher disability ( $<0.001$ for both ADL and IADL), burden and severity of comorbidities ( $p<0.05$ and $p<0.01$ for CIRS-C and CIRS-S, respectively), cognitive impairment (SPMSQ, p<0.01) and malnutrition (MNA, p<0.05). Moreover, the overall prevalence of delirium (either hyperor hypo-kinetic) was significantly higher in patients of the IA group $(6.4 \%$ positive CAM vs $3.1 \%$ in the SA group, $\mathrm{p}<0.05)$.

\section{PPR Risk Factors}

Stepwise regression analysis was performed in order to find potentially predictive factors of early readmission. PPR was the dependent variable and age, sex, each geriatric scale score

Table I Demographic Data And Length Of Hospital Stay (days) According To The PPRG (Potentially Preventable Readmissions Grouping) Software Classification

\begin{tabular}{|l|l|l|l|l|}
\hline & Total & SA & IA & PPR \\
\hline DRG codes & 1640 & $1263(77.0 \%)$ & $17 \mid(10.4 \%)$ & $206(12.6 \%)$ \\
Males & $738(45.0 \%)$ & $563(44.6 \%)$ & $78(45.6 \%)$ & $94(45.6 \%)$ \\
Females & $902(55.0 \%)$ & $700(55.4 \%)$ & $93(54.4 \%)$ & $112(54.4 \%)$ \\
Age (mean \pm SD) & $84.6 \pm 7.4$ & $84.0 \pm 7.4$ & $84.4 \pm 7.2$ & $84.9 \pm 7.1$ \\
Length of hospital stay (median and range) & $5(1-32)$ & $5(4-6)$ & $6(2-14)^{*}$ & $6(2-14)^{*}$ \\
\hline
\end{tabular}

Note: $* P<0.05$ Vs SA.

Abbreviations: DRG, Diagnosis-Related Group; SA, Stand-alone Admissions; IA, Index Admissions; PPR, Potentially Preventable Readmissions. 

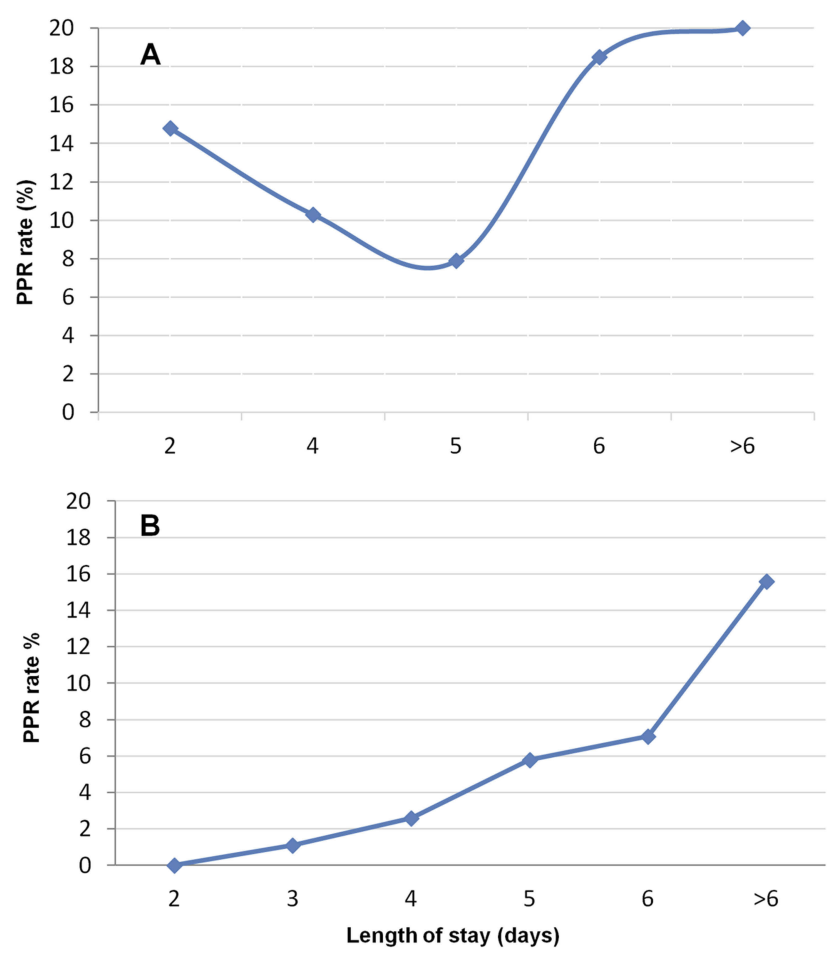

Figure $\mathbf{2}$ Rate of potentially preventable readmissions. (A) Cardiovascular diseases; (B) Other diseases.

Abbreviations: PPR, Potentially Preventable Readmissions; DRG, Diagnosis Related Group.

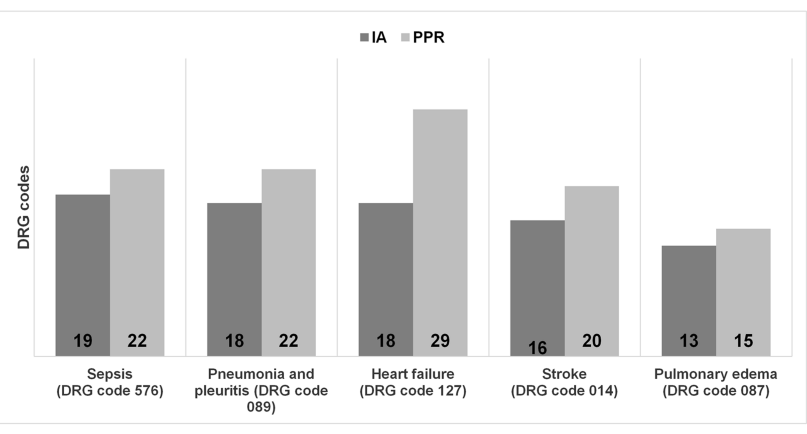

Figure 3 More frequently DRG codes in IA and PPR groups.

Abbreviations: IA, Index Admission PPR, Potentially Preventable Readmissions DRG, Diagnosis-Related Group.

and the five most frequent DRG codes were included as covariates. At the end of the analysis, among geriatric scores the lowest tertile of degree of disability (ADL score $0-1$ ) emerged as the most significant independent risk factor for early re-hospitalization (OR 2.06 [95\% CI 1.34-3.16], $\mathrm{p}<0.001)$ followed by the highest quartile of the severity of comorbidity (CIRS-S >2.38) (OR 1.73 [95\% CI 1.02-2.93], $\mathrm{p}<0.05$ ) while, only the DRG code 576 (sepsis) maintained a significant independent association with PPR (OR 2.56 [95\% CI 1.31-5.00], $\mathrm{p}=0.01$ ) (Table 3). Accordingly, we can define
Table 2 Comprehensive Geriatric Assessment Of Patients In IA And SA Groups

\begin{tabular}{|c|c|c|c|}
\hline & SA $(n=\mid 263)$ & IA $(n=|7|)$ & $\mathbf{p}$ \\
\hline Females (\%) & 56.9 & 54.6 & ns \\
\hline Age (mean $\pm S D)$ & $84.0 \pm 7.4$ & $84.4 \pm 7.2$ & ns \\
\hline CIRS-S (mean \pm SD) & $2.0 \pm 0.6$ & $2.2 \pm 0.6$ & $<0.01$ \\
\hline CIRS-C (mean $\pm S D)$ & $5.0 \pm 1.9$ & $5.5 \pm 1.8$ & $<0.05$ \\
\hline $\mathrm{ADL}($ mean $\pm S D)$ & $3.1 \pm 2.5$ & $2.2 \pm 2.4$ & $<0.001$ \\
\hline IADL (mean $\pm S D)$ & $2.8 \pm 2.8$ & $1.8 \pm 2.3$ & $<0.001$ \\
\hline SPMSQ $($ mean $\pm S D)$ & $4.3 \pm 3.5$ & $5.2 \pm 3.5$ & $<0.01$ \\
\hline Malnutrition [n (\%)] & $316(25.0)$ & $7 \mid(4 I .5)$ & $<0.05$ \\
\hline Delirium [n (\%)] & $39(3.1)$ & II (6.4) & $<0.05$ \\
\hline
\end{tabular}

Abbreviations: IA, Index Admissions; SA, Stand-alone Admissions; CIRS-S, Cumulative IIIness Rating Scale - Severity; CIRS-C, Cumulative Illness Rating Scale Comorbidity; ADL, Activities of Daily Living; IADL, Instrumental Activity of Daily Living; SPMSQ, Short Performance Mental Status Questionnaire; ns, not significant.

Table 3 Independent Risk Factors For Potentially Preventable Readmissions

\begin{tabular}{|c|c|c|c|c|}
\hline \multirow{2}{*}{ Readmission Phenotype } & \multirow[t]{2}{*}{ Odds Ratio } & \multicolumn{2}{|c|}{ Cl $95 \%$} & \multirow[b]{2}{*}{$\mathbf{p}$} \\
\hline & & Min & Max & \\
\hline \multicolumn{5}{|l|}{ ADL } \\
\hline [5-6] & I & & & \\
\hline$[2-4]$ & 1.47 & 0.91 & 2.37 & NS \\
\hline$[0-1]$ & 2.06 & 1.34 & 3.16 & $<0.001$ \\
\hline \multicolumn{5}{|l|}{ CIRS-S } \\
\hline$\leq 1.84$ & I & & & \\
\hline $1.84-2.08$ & I.II & 0.65 & 1.91 & NS \\
\hline $2.08-2.38$ & 1.34 & 0.78 & 2.32 & NS \\
\hline$>2.38$ & 1.73 & 1.02 & 2.93 & $<0.05$ \\
\hline Sepsis (DRG 576) & 2.56 & 1.31 & 5.00 & 0.01 \\
\hline
\end{tabular}

Abbreviations: ADL, Activities of Daily Living; CIRS-S, Cumulative Illness Rating Scale-Severity; DRG, Diagnosis Related Group; NS, not significant.

two extreme risk phenotypes of PPR: "lowest risk phenotypes" in the case of ADL score 5-6, CIRS-S $<1.84$, female sex, DRG other than sepsis (6.7\% risk of readmission); "highest risk phenotypes" in cases of ADL score 0-1, CIRS-S >2.38, male sex, and diagnosis of sepsis (30.1\% risk of readmission).

\section{Discussion}

The overall early readmission rate of our cohort of geriatric patients (mainly oldest old) hospitalized for acute disease was slightly less than that generally reported by the literature (11.9\% vs $14-20 \%)$, possibly because in the current study only PPR and not all early readmissions were analyzed. ${ }^{8-11}$ Interestingly, $17 \%$ of the IA resulted in multiple PPR and the DRG code 127 (acute HF) generated the highest number of multiple readmissions (1.61 PPR by each IA). 
As a whole, five DRG codes accounted for almost $40 \%$ of all PPR but, by multivariate regression analysis, only the DRG code 576 (sepsis) along with the presence of severe disability (ADL score 0-1) and a high degree of comorbidities (CIRS-S score>2.38) emerged as significant independent risk factors for PPR. Accordingly, a recent review on long-term outcome after sepsis showed high rates of weakness, cognitive impairment, hospital readmission, and late death in sepsis survivors. ${ }^{12}$ Patients' age did not affect the rate of PPR, possibly since the age distribution of the cohort was limited and most of the patients were ultra-octogenarian. Interestingly, age did not represent a risk factor for early readmission also in a surgical cohort with only $14.9 \%$ of the patients older than 65 years. ${ }^{13}$ However, it is worth noting that the overall prevalence of early readmissions was small $(8.2 \%)$ and a large number of readmissions were mainly due to confounding issues of substance abuse or homelessness. ${ }^{10}$ Although the substantially shorter (5 days) median length of hospital-stay of our cohort, it resulted in significantly longer in both IA and PPR than in SA patients $(p<0.05)$, suggesting that the reduction of the length of hospital stay may reduce, rather than increase, the risk of PPR. However, a peculiar figure was observed in the case of acute HF (DRG code 127), characterized by a nonlinear U-shaped PPR rate according to the length of hospital stay (with the nadir at 5 days), while in all the other DRG codes, PPR rate progressively increased with increasing length of stay. This finding is in line with the results of a multicenter cohort study of 58,230 older HF patients (median age 80 years) conducted in Canada between April 2003 and March 2012. ${ }^{14}$ A short length of stay after hospitalization for HF was associated with increased rates of cardiovascular and HF readmissions but lower rates of noncardiovascular readmissions, while a long length of stay was associated with increased rates of all types of readmission and mortality. ${ }^{14}$ The underlying reasons for the peculiar trend observed in our patients with HF are complex. The possible role of non-cardiovascular diseases, as reported in the literature, ${ }^{15,16}$ is unlikely, given the high burden of comorbidities of most of our patients, regardless of HF. On the other hand, increased risk of readmission with a short length of stay may be explained by persistent congestion, or not optimized medications and transitional care. ${ }^{17}$ This suggests that case-mix severity and factors such as inadequate transitional care and coordination of services, ${ }^{18,19}$ and lack of access to outpatient services might be responsible for repeated hospitalization. Accordingly, a recent retrospective cohort study from Ontario, Canada, showed that among patients who received emergency care for $\mathrm{HF}$, obtaining follow-up within
1 week of discharge from the emergency department may be associated with reduced rate of subsequent admission and death, regardless of the length of hospital stay. ${ }^{20}$ Besides these considerations, diverse factors may lead to the continuing higher rate of rehospitaliation with increasing length of stay, as observed in all the other DRG codes $(087,576,089$, 014). Indeed, in very old patients, frail phenotype, high burden of comorbidities and various degrees of disability actually worsen their clinical condition, increasing the length of hospital stay with associated immobilization, malnutrition, sleep deprivation, and risk of delirium. ${ }^{21,22}$ Thus, good clinical practice implies that, in complex and frail older patients, shorter hospital stay with preservation of psychophysical autonomy may prevail over complete cure of the underlying acute disease. Obviously, adequate transitional care and coordination of services are also necessary. Accordingly, disability emerged as the more relevant risk factor for PPR in our cohort. Both ADL and IADL score results were notably reduced in the IA compared to the SA group, indicating that a functionally impaired subject is likely to be readmitted early on. Moreover, we documented a significant higher prevalence of malnutrition in patients of the IA group compared to the SA group ( $41.5 \%$ vs $25 \%$, respectively, $\mathrm{p}<0.05$ ). Only a few studies have focused on malnutrition, which was linked to longer length of stay, worse clinical outcome and higher readmission rate. ${ }^{23,24}$ It is noteworthy that poor nutritional status is part of the frail phenotype, exposing patients to disability, hospitalizations, and death. ${ }^{25}$ According to our findings, a prospective multi-center study demonstrated that markers of frailty or severe disability assessed by comprehensive geriatric assessment were the most important predictors of early readmission. ${ }^{6}$ Our results, although in a smaller cohort, are in line with a recent study conducted on a geriatric community-dwelling population admitted to a Medicare hospital over a period of 10 years. ${ }^{9}$

Besides disability and malnutrition, cognitive impairment correlated with the risk of PPR, allows us to shape two extreme phenotypes, by calculating the marginal probability of early readmission: one at the highest risk $(30.1 \%$, oldestold malnourished patient with impaired cognition, important disability and severe comorbidities) opposed to one at the lowest risk (6.7\%). Thus, an implemented strategy to identify patients more at risk appears crucial, especially considering the heavy consequences of PPR. Minimizing delirium, immobility and distress, together with correct therapy and discharge plans, should be the aim of in-hospital care, and taking care of new disabilities and medical deterioration should be the post-discharge focus. ${ }^{12}$ Our results, although 
in a small cohort of very old patients, underlie the role of comprehensive geriatric assessment as the cornerstone to identify patients at higher risk of PPR. This kind of workup should lead to a careful discharge plan, adequately supporting disabled patients, and follow-up with General Practitioners and outpatient clinics. As already demonstrated, ${ }^{26}$ a trained nurse or a geriatrician in the ED, providing a first geriatric evaluation and managing the discharge, could reduce PPR, saving frail older patients from a potentially more dangerous hospital environment. The main strength of our study is the comprehensive multi-dimensional geriatric assessment provided, homogenous among the patients, administered by consistently trained personnel. A limitation of the study is the relatively small cohort and, larger, multicentre studies are necessary to confirm these findings, using the same geriatric scales' battery. The selection of the cohort, limited to the subjects admitted from the ED and excluding patients with underlying disease certainly requiring repeated admissions, could be considered a limitation of the study. On the other hand, this represents a suitable sub-population where strategies for preventing readmissions should be targeted.

\section{Conclusion}

Early hospital readmissions represent a social, medical and economic challenge worldwide. Given the rising burden of (end-stage) chronic diseases due to the increasing aging population, it is crucial to address PPR, especially in the oldest-old population. The measures that clearly need to be improved are the adequacy of the treatment during hospitalization (especially in cases of sepsis and acute HF) as well as the setting of a comprehensive discharge plan, accounting for the disability and comorbidity of the patient, which represent strong, independent predictive risk factors for PPR. The current study also provides evidence to support early follow-up for these patients, requiring a transition between emergency and longitudinal care, via collaboration between administrators, clinicians and information technology specialists.

\section{Abbreviations}

DRG, Diagnosis-Related Group; SA, Stand-alone Admissions; IA, Index Admissions; PPR, potentially preventable readmissions; APR-DRG, All-Patient-RefinedDiagnosis-Related Group, CIRS, Cumulative Illness Rating Scale; ADL, Activities of Daily Living; IADL, Instrumental Activities of Daily Living; SPMSQ, Short Portable Mental Status Questionnaire; MNA, Mini Nutritional Assessment;
CAM, Confusion Assessment Method; ED, emergency department; HF, heart failure.

\section{Acknowledgments}

We wish to thank Drs Di Maio Alessandra, Vio Gabriele and Filippi Matteo, who helped in using the $3 \mathrm{M}^{\mathrm{TM}}$ software and Dr Paola De Feo, for her assistance in collecting the data.

\section{Author Contributions}

All authors contributed toward data analysis, drafting and revising the paper, gave final approval of the version to be published and agree to be accountable for all aspects of the work.

\section{Disclosure}

The authors report no conflicts of interest in this work.

\section{References}

1. Bianco A, Mole A, Nobile CG, et al. Hospital readmission prevalence and analysis of those potentially avoidable in Southern Italy. PLoS One. 2012;7(11):e48263. doi:10.1371/journal.pone.0048263

2. Lapointe-Shaw L, Mamdani M, Luo J, et al. Effectiveness of a financial incentive to physicians for timely follow-up after hospital discharge: a population-based time series analysis. CMAJ. 2017;189 (39):1224-1229. doi:10.1503/cmaj.170092

3. Zhou H, Della PR, Roberts P, Goh L, Dhaliwal SS. Utility of models to predict 28-day or 30-day unplanned hospital readmissions: an updated systematic review. BMJ Open. 2016;6(6):e011060. doi:10.1136/bmjopen-2016-011060

4. Henderson J, Goldacre MJ, Graveney MJ, et al. Use of medical record linkage to study readmission rates. $\mathrm{Br}$ Med J. 1989;299 (6701):709-713. doi:10.1136/bmj.299.6701.709

5. Holloway JJ, Thomas JW, Shapiro L. Clinical and sociodemographic risk factors for readmission of medicare beneficiaries. Health Care Financ Rev. 1988;10(1):27-36.

6. Laniece I, Couturier P, Drame M, et al. Incidence and main factors associated with early unplanned hospital readmission among French medical inpatients aged 75 and over admitted through emergency units. Age Ageing. 2008;37(4):416-422. doi:10.1093/ageing/afn093

7. Garcia-Perez L, Linertova R, Lorenzo-Riera A, et al. Risk factors for hospital readmissions in elderly patients: a systematic review. QJM. 2011;104(8):639-651. doi:10.1093/qjmed/hcr070

8. Braes T, Moons P, Lipkens P, et al. Screening for risk of unplanned readmission in older patients admitted to hospital: predictive accuracy of three instruments. Aging Clin Exp Res. 2010;22(4):345-351.

9. Greysen SR, Cenzer IS, Auerbach AD, et al. Functional impairment and hospital readmission in medicare seniors. JAMA Intern Med. 2015;175(4):559-565. doi:10.1001/jamainternmed.2014.7756

10. Fernandez-Gasso L, Hernando-Arizaleta L, Palomar-Rodríguez JA, et al. Trends, causes and timing of 30-day readmissions after hospitalization for heart failure: 11-year population-based analysis with linked data. Int J Cardiol. 2017;248:246-251. doi:10.1016/j.ijcard. 2017.07.094

11. Krumholz HM, Wang K, Lin Z, et al. Hospital-readmission risk isolating hospital effects from patient effects. $N$ Engl J Med. 2017;377(11):1055-1064. doi:10.1056/NEJMsa1702321 
12. Royer S, DeMerle KM, Dickson RP, et al. Shorter versus longer courses of antibiotics for infection in hospitalized patients: a systematic review and meta-analysis. J Hosp Med. 2018;13(5):336-342. doi: $10.12788 /$ jhm. 2905

13. McIntyre LK, Arbabi S, Robinson EF, et al. Analysis of risk factors for patient readmission 30 days following discharge from general surgery. JAMA Surg. 2016;151(9):855-861. doi:10.1001/jamasurg. 2016.1258

14. Sud M, Yu B, Wijeysundera HC, et al. Associations between short or long length of stay and 30-day readmission and mortality in hospitalized patients with heart failure. JACC Heart Fail. 2017;5(8):578588. doi:10.1016/j.jchf.2017.03.012

15. Cotter G, Davison BA, Milo O, et al. Predictors and associations with outcomes of length of hospital stay in patients with acute heart failure: results From VERITAS. J Card Fail. 2016;22(10):815-822. doi:10.1016/j.cardfail.2015.12.017

16. Whellan DJ, Zhao X, Hernandez AF, et al. Predictors of hospital length of stay in heart failure: findings from get with the guidelines. J Card Fail. 2011;17(8):649-656. doi:10.1016/j.cardfail.20 11.04 .005

17. Naylor MD, Bowles KH, McCauley KM, et al. High-value transitional care: translation of research into practice. J Eval Clin Pract. 2013;19(5):727-733. doi:10.1111/j.1365-2753.2011.01659.x

18. Naylor MD, Aiken LH, Kurtzman ET, et al. The care span: the importance of transitional care in achieving health reform. Health Aff (Millwood). 2011;30(4):746-754. doi:10.1377/hlthaff.2011.0041
19. Boutwell AE, Johnson MB, Rutherford P, et al. An early look at a four-state initiative to reduce avoidable hospital readmissions. Health Aff. 2011;30(7):1272-1280. doi:10.1377/hlthaff.2011.0111

20. Atzema CL, Austin PC, Yu B, et al. Effect of early physician follow-up on mortality and subsequent hospital admissions after emergency care for heart failure: a retrospective cohort study. CMAJ. 2018;17:190.

21. Maeda K, Koga T, Akagi J. Nutritional variables predict chances of returning home and activities of daily living in post-acute geriatric care. Clin Interv Aging. 2018;13:151-157. doi:10.2147/CIA.S154129

22. Wesselius HM, van Den Ende ES, Alsma J, et al. Quality and quantity of sleep and factors associated with sleep disturbance in hospitalized patients. JAMA Intern Med. 2018;178:1201. doi:10. 1001/jamainternmed.2018.2669

23. Charlton K, Nichols C, Bowden S, et al. Poor nutritional status of older subacute patients predicts clinical outcomes and mortality at 18 months of follow-up. Eur J Clin Nutr. 2012;66(11):1224-1228. doi:10.1038/ejen.2012.130

24. Sharma Y, Miller M, Kaambwa B, et al. Malnutrition and its association with readmission and death within 7 days and 8-180 days postdischarge in older patients: a prospective observational study. BMJ Open. 2017;7(11):e018443.

25. Cesari M, Calvani R, Marzetti E. Frailty in older persons. Clin Geriatr Med. 2017;33(3):293-303. doi:10.1016/j.cger.2017.02.002

26. Hwang U, Dresden SM, Rosenberg MS, et al. Geriatric emergency department innovations: transitional care nurses and hospital use. $J$ Am Geriatr Soc. 2018;66:459-466. doi:10.1111/jgs.2018.66.issue-3
Clinical Interventions in Aging

\section{Publish your work in this journal}

Clinical Interventions in Aging is an international, peer-reviewed journal focusing on evidence-based reports on the value or lack thereof of treatments intended to prevent or delay the onset of maladaptive correlates of aging in human beings. This journal is indexed on PubMed Central, MedLine, CAS, Scopus and the Elsevier

\section{Dovepress}

Bibliographic databases. The manuscript management system is completely online and includes a very quick and fair peer-review system, which is all easy to use. Visit http://www.dovepress.com/ testimonials.php to read real quotes from published authors. 